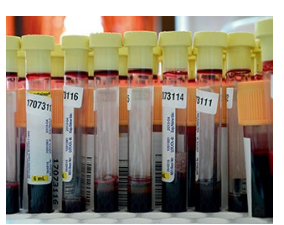

Liquid biopsy tests for cancer march on $\mathrm{p} 972$

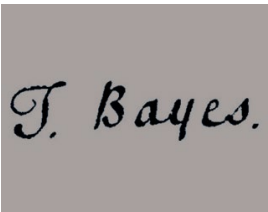

I-SPY clinical trials hit the gas $\mathrm{p} 975$

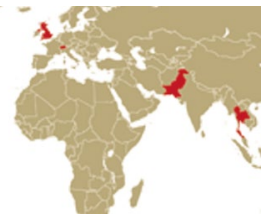

Biotech news from around the world p977

\title{
Pursuit of tumor-infiltrating lymphocyte immunotherapy speeds up
}

\author{
Mounting evidence of efficacy in metastatic breast cancer and other epithelial tumors is driving clinical and \\ commercial investment in tumor-infiltrating lymphocytes.
}

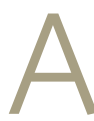

t the beginning of July, Iovance Biotherapeutics announced that the US Food and Drug Administration may consider its ongoing phase 2 study of tumor-infiltrating lymphocyte (TIL) therapy $\mathrm{LN}-145$ sufficient to support registration as a treatment for advanced cervical cancer. This therapy-which involves extracting T cells from a patient's tumor, expanding them ex vivo and reinjecting them-is one of several TIL treatments showing encouraging results in the clinic. Other groups are also joining the fray, pursuing TILs for various solid cancers (Table 1).

Earlier in the summer, at the annual meeting of the American Society of Clinical Oncology (ASCO), surgical oncologist Stephanie Goff of the US National Cancer Institute (NCI) presented a case report of a 49-year-old woman with advanced breast cancer who was treated with her own T cells, obtained from the tumor. After the cells were expanded ex vivo, the patient was infused with 80 billion cells. Her tumors completely disappeared, and she has remained tumor-free for 42 months. "TILs are capable of eliminating the last cancer cell," Goff said at ASCO.

TIL therapy is not a new modality. NCI surgeon Steve Rosenberg began treating melanoma patients with TILs back in 1986 . But the approach has always faced two major limitations: growing TILs is a tricky, timeconsuming and individualized laboratory procedure; and TILs showed little activity in any cancer except melanoma. Researchers are now achieving broader success and challenging these limitations. As the breast cancer case report dramatized, Rosenberg's group (which includes Goff) is achieving some success in such epithelial tumors. And, now that companies have commercialized chimeric antigen receptor T cells (CAR-Ts), groups pursuing TILs are eager to follow suit, expanding patient TILs at centralized

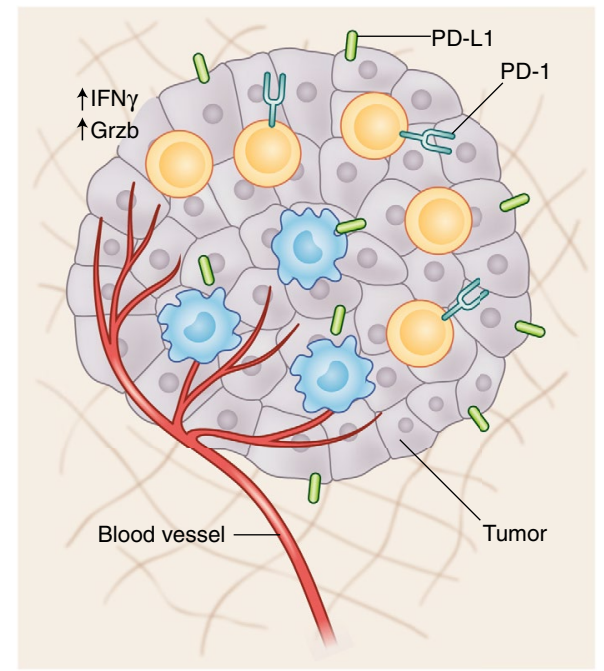

Tumor-infiltrating lymphocytes (yellow) react against tumor cells (purple). Companies are now commercializing ex vivo expansion of TILs for return to patients. PD-L1, programmed cell death ligand 1; IFN, interferon; Grzb, granzyme B. Credit: Adapted with permission from

Nature Medicine 24, 541-550, 2018

manufacturing sites before freezing and delivering them to hospitals to give to patients. "There is so much we can do in this space," says Maria Fardis, the CEO of Iovance Biotherapeutics, which leads the TIL field.

TILs, unlike CAR-T cells and T cells with engineered T cell receptors (Nat. Biotechnol. $36,215-219,2018)$, are not genetically modified. The basic method: technicians chop up a surgically resected tumor and place it in culture medium with growth factors, typically interleukin-2 and a CD3 agonist to stimulate $\mathrm{T}$ cell proliferation. "We let the $T$ cells spill out of the tumor cells and start growing," explains Chantale Bernatchez, research director at the MD Anderson
Cancer Center TIL laboratory in Houston. The expansion process typically requires five to six weeks to grow enough cells-up to 50 billion-to reinfuse into the patient.

To help condition the body for the TIL transplant, patients receive a reversible lymphodepleting chemotherapy regimen before infusion. This step eliminates competition for growth-promoting cytokines and removes suppressor cells like $\mathrm{T}$ regulatory cells and myeloid-derived suppressor cells. Lymphodepletion "gives a head start to the T cells that we infuse," says Bernatchez. Such T cells can be remarkably long-lived; Bernatchez's group has detected them in large numbers in successfully treated patients five years after infusion. Researchers have also improved their ability to harvest and expand T cells, with the NCI and Iovance reporting success in harvesting TILs from over $90 \%$ of patients with bulky tumors.

Iovance first sought to commercialize TILs back in 2011, when it licensed Rosenberg's TIL technology in melanoma. The company, then called Genesis Biopharma, later renamed Lion Biotechnologies and now Iovance, launched its first phase 2 melanoma trial in 2015 . The protocol was later amended to include cryopreserved cells and a shortened manufacturing process.

Results for that open-label melanoma trial, reported at ASCO, were for Iovance's LN-144 cryopreserved TIL product. All patients enrolled in the study had previously been treated with therapy targeting programmed cell death receptor 1 (PD-1) but had progressed. With LN-144 treatment, 25 of 66 patients had an objective response by standard radiological criteria, including two complete responses. Although most participants experienced severe side effects (including one death from intraabdominal hemorrhage), attributable 


\section{Fight over CRISPR IP flares up}

The dispute over who was first to invent CRISPR-Cas9 genome editing in eukaryotic cells has resumed with the US Patent and Trademark Office (USPTO) declaring an interference between the University of California (UC) and the Broad Institute of MIT and Harvard. The patent office's decision came in June, just nine months after a federal court upheld a no-interference judgement that gave the Broad control of key CRISPR intellectual property. The new interference case involves 13 of the Broad's 15 patents and one application, and 10 patent applications submitted by UC Berkeley and partners. It is a signal that the USPTO has determined that one or more of Berkeley's patent applications are substantially the same as previously issued Broad patents. Since the federal court's September 2018 ruling, which declared no interference and appeared to be a win for Broad and Harvard, UC Berkeley has been granted six CRISPR patents in the United States. These include a foundational patent that covers systems and methods for the use of single-molecule guide RNAs combined with the Cas 9 protein to modify target DNA in any setting, including in vitro and in cells. In addition, in May the Broad Institute had its third European CRISPR patent revoked by the European Patent Office for lacking in inventive step. UC Berkeley has maintained a dominant CRISPR patent position in Europe, while the Broad has held the upper hand in the United States. However, the newly filed interference could upend the current balance of power. "The initiation of this interference proceeding highlights that previous decisions involving the Broad did not determine who was the first to invent this technology, and it lays out a pathway for resolving this important issue," said Eldora Ellison, lead patent strategist on CRISPR matters for UC Berkeley.

Published online: 4 September 2019 https://doi.org/10.1038/s41587-019-0256-2

\footnotetext{
C "It's hard to say for sure what we can achieve in the next few decades, but I think with CRISPR we have a chance to catch up to climate change." Yiping Qi, a plant genome engineer at the University of Maryland, comments on how CRISPR could accelerate the introduction of plants that withstand droughts, heat waves, megafloods and other expected climate changes. (Wired, 8 August 2019)
}

mainly to the lymphodepletion regimen and the interleukin-2 infused to boost TIL survival and growth, these resolved two weeks after infusion. The $38 \%$ response rate was, "an excellent result in this population," commented immunologist Ignacio Melero of the Clinica Universidad de Navarra in Madrid, Spain at ASCO. At an 8.8-month follow up, 17 of 25 responders had an ongoing response, suggesting durable effects.

"My concern is that with more follow up their duration of response may go down," says Bernatchez. "But right now they're showing pretty good numbers."

Iovance is now enrolling a new melanoma cohort using its secondgeneration TIL protocol, which cuts the previous five- to six-week manufacturing time to 22 days. Although Iovance has not disclosed the details for this improved protocol, Iovance's Fardis expects trial enrollment to be complete by the first quarter of next year, with a possible Biologic License Application filing by year's end.

At ASCO, Iovance also reported ongoing results for LN-145 in its open-label phase 2 trial for metastatic cervical cancer. So far 12 of 27 patients have experienced an objective response, including three complete responses. Ten of those whose disease responded had an ongoing response at a median follow up time of 7.4 months. That's impressive, says Bernatchez, but "I'd be careful with those high response rates," she adds. "I won't believe it until I see that the response rates are really durable." Given the FDA's willingness to consider the complete data, Fardis says Iovance may file its Biologic License Application for cervical cancer by late 2020 .

For companies, the number of TILs needed for infusion poses a greater logistical challenge than for CAR-Ts. A single CAR-T dose is around 100 million cells. "TIL [therapy] (as best we know now) requires products with 100 times that many cells, on the order of 30-50 billion," writes Goff in an e-mail. "Cryopreserving that many cells and maintaining viability of the thawed product is certain to be a challenge."

Even with manufacturing obstacles cleared, there's still a huge gap between success in melanoma and cervical cancer, on the one hand, and other cancers on the other. This may be because breast, colon, ovary, prostate and pancreatic cancers are poorly immunogenic. Of the harvested TILs, only a fraction are reactive against the tumors, so giving patients their own heterogeneous TILs appears futile. "Unselected TILs in our hands certainly did not work in patients with epithelial cancers," says Goff. "We had zero responses."

To boost tumor killing, Rosenberg's group selects only tumor neoantigen-reactive
T cells among patients' TILs. First they isolate tumor DNA or RNA, and use whole exome or transcriptome sequencing to identify all amino acid-altering mutations by comparison with sequenced normal tissue. Then the NCI team designs gene constructs containing sequences for all the mutant peptides that could be displayed on the major histocompatibility complex (MHC) of antigen-presenting cells. They then string sequences together into 'tandem minigenes' and insert them into the patient's own antigen-presenting cells, to test patient $\mathrm{T}$ cells for reactivity. The reactive $\mathrm{T}$ cells, expanded in culture, are reinfused into the patient.

\section{"At some point we will get there... right now, we should rely on the patient's immune system, which has selected the right TILs:"}

The main advantage of the NCI selection method is exquisite specificity for a given patient's tumor. With the tandem mini-genes approach, the NCI has achieved a roughly $15 \%$ clinical response rate across metastatic epithelial cancers. This includes the breast cancer patient with the ongoing complete response that Goff highlighted at ASCO.

But to design and construct the tandem minigenes alone takes up to a month. The whole process, from tumor excision to product readiness, takes a median of ten weeks, says Goff. That's not fast enough for most cancer patients, says Bernatchez. "I think [the NCI approach] has value, but unless it's highly streamlined, it's going to be very difficult to apply to large patient populations," she says. "You need to get it at least under one month."

Iovance's Fardis agrees. "We have to think about a process that can be industrialized," she says. Iovance shaved its process down to 22 days partly by omitting the neoantigen-reactive T-cell selection step. Such selection isn't necessary, Fardis says. "Science right now is not ready to collect all the known neoantigens," she says. (The NCI system misses post-translational modifications and edited RNA.) "At some point we will get there ... right now, we should rely on the patient's immune system, which has selected the right TILs," although in diluted form.

So for now, both Iovance and MD Anderson are using bulk, unselected TILs. But they're exploring markers on T cells that could enable selection of tumor-reactive $\mathrm{T}$ cells, as well as ways of making $\mathrm{T}$ cells more potent. Iovance hopes to have a selective T-cell product in the clinic by 2020 . "I don't 
Table 1 | Selected clinical trials of TIL and related adoptive cell therapy

\begin{tabular}{|c|c|c|c|}
\hline Company or institution & Agent & Indication & Stage \\
\hline lovance Biotherapeutics & Lifileucil (LN-144) & Melanoma & Pivotal phase 2 \\
\hline lovance Biotherapeutics & LN-145 & Cervical, head and neck cancers & Phase 2 \\
\hline Netherlands Cancer Institute & TIL & Melanoma & Phase 3 \\
\hline US National Cancer Institute & Neoantigen-specific TIL & $\begin{array}{l}\text { Melanoma, breast, colorectal, ovarian, pancreatic } \\
\text { cancers; glioblastoma, }\end{array}$ & Phase 2 \\
\hline MD Anderson Cancer Center & MDA-TIL & Ovarian, pancreatic cancers; osteosarcoma, & Phase 2 \\
\hline WindMIL Therapeutics & Marrow-infiltrating lymphocytes & Multiple myeloma & Phase 2 \\
\hline H. Lee Moffitt Cancer Center & TIL & Non-small-cell lung carcinoma & Phase 1 \\
\hline
\end{tabular}

think it's this binary landscape where it's either selection or un-selection," Fardis says.

One startup, however, considers neoantigen-specific TILs very feasible today. Myst Therapeutics, founded by biomedical engineer Timothy Langer, is fully committed to TIL selection. Langer, who previously worked at Kite Pharma (now owned by Gilead) designing the Yescarta (axicabtagene ciloleucel) CAR-T product and later led development of engineered $\mathrm{T}$ cell receptors targeting neoantigens, founded Myst in January and has secured a license from the NCI. "Four or five years ago, it was perceived that even cell therapy was too difficult to do," Langer says. "Having been through that experience and looking at this particular problem, I see it as very doable, as an execution issue." Manufacturing time must be shortened, Langer says. But unlike many ideas in biotech, TIL selection in solid tumors "is one that actually has some proof of concept, or at least anecdotal proof of concept, where it has worked." Myst has raised single-digit millions from angel investors and others, and is leasing lab space at the University of California, Los Angeles.

Other biotechs and academic groups have entered the TIL space. "A lot of companies are interested in what TIL therapy may bring to the field," says Bernatchez, who says her consulting work load has grown quickly just in the past few months. TILs' curative potential is a big draw. "In melanoma, when you have a complete response with TILs that's ... over a year, then usually the patient is cured," Bernatchez says. Of the NCI's 46 complete responders in melanoma, says Goff, only two have relapsed. Non-melanoma solid tumors will be harder, and success or failure there will determine whether TILs remain a niche treatment or evolve into a foundational cancer therapy.

\section{Ken Garber}

Ann Arbor, Michigan, USA

Published online: 7 August 2019

https://doi.org/10.1038/d41587-019-00023-6

\section{PODCAST}

\section{First Rounders: Nina Tandon}

Nina Tandon is cofounder and CEO of EpiBone. Her First Rounders conversation covers growing up on Roosevelt Island in New York City, witnessing September 11, and tackling her company's first phase 1 trial. https://www.nature.com/nbt/podcast

Published online: 4 September 2019

https://doi.org/10.1038/s41587-019-0243-7

"It's like saying a skateboard needs to have a sticker to say it can't be used on the freeway. It doesn't make any sense." Josiah Zayner, biohacker and founder of Odin, a company providing do-it-yourself kits for genetic engineers, known for injecting himself with CRISPR on social media, comments on a new law in California prohibiting the sale of CRISPR kits unless they warn against self-administration. (MIT Technology Review, 9 August 2019)
"Manufacturing pharmaceuticals is difficult, painstaking, and dangerous. If you get the dosing or the strength on insulin wrong, it's death. If you let contaminants into the insulin, it's possible death. If your insulin breaks down too quickly in storage, it's death." Stanford's Hank Greely comments on the 'Open Insulin Project', run by a group of biohackers in Oakland, California, to create a low-cost insulin. (Dw.com, 24 May 2019)

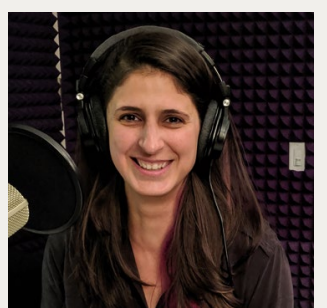

"Consumers might be surprised by some of the uses that their data are being put toward, and they might be concerned about some of the third parties their data are being shared with." James Hazel of Vanderbilt's Center for Genetic Privacy and Identity in Community Settings, in light of Congress considering legislation on digital data privacy that could restrict what direct-to-consumer genomics companies can do with the data they collect. (STAT, 9 August 2019) 